\title{
Self-interactions in the space-time of a scalar-tensor cosmic string
}

\author{
I. V. L. Costa and F. A. Oliveira \\ Instituto de Física, Universidade de Brasília, \\ CEP: 70910-900, Brasília, DF, Brazil \\ M. E. X. Guimarães \\ Departamento de Matemática, Universidade de Brasília \\ CEP: 70910-900, Brasília, DF, Brazil \\ Fernando Moraes
}

Departamento de Física, Universidade Federal da Paraíba, 58051-970 João Pessoa, PB, Brazil

\begin{abstract}
We study the effect of the geometry and topology of a scalar-tensor cosmic string space-time on the electric and magnetic fields of line sources. It is shown that the dilatonic coupling of the gravity induces effects along the string comparable to a current flow allowing for forbidden regions near the string.
\end{abstract}

PACS numbers: 98.80.Cq;11.27.+d

\section{INTRODUCTION}

It is a well known fact [1, 2] that a point charge in a static gravitational field experiences an electrostatic force due to the deformation of its electric field lines produced by the geometry of space-time. The net effect is a self-force on the charge. The study of self-forces on electric charges in the presence of topological defects started when Linet [3] and Smith [4] found the electrical self-force on a point charge in the presence of a cosmic string. Bezerra de Mello et al. [5] found both the electric self-force on a line of charge and the magnetic self-force on an electrical current parallel to a cosmic string.

All the above-mentioned results have been obtained in the framework of Einstein's gravity. Here, we intend to generalize these results considering a scalar-tensor gravity. The motivation for this relies on the fact that, theoretically, the possibility that gravity might not be fundamentally Einsteinian is gathering credence. This is in part a consequence of superstring theory [6], which is consistent in ten dimensions (or M-theory in eleven dimensions), but also the more phenomenological recent developments of "braneworld" scenarios 7, 8] have motivated the study of other gravitational theories in fourdimensions. In fact, the origin of the (gravitational) scalar field can be many: the scalar field arising from the size of the compactified internal space in the KaluzaKlein theory; the zero mode (dilaton field) described by a symmetric second-rank tensor behaving as space-time metric at low energy level in the closed string theory; the scalar field in a brane world scenario; and more [9]. In any case, clearly, if gravity is essentially scalar-tensorial there will be direct implications on observed effects both in the small scale scenarios of alternative theories of gravity 10, 11, 12, 13 and in the large scale cosmological scenarios from modified gravity 14.

It is our interest here to consider small-scale effects and to show these modifications to the case of a scalar- tensor cosmic string. In this work we find the self-energy and self-force on a line source in the presence of a scalartensor cosmic string. We look both at the case of the source being a line with uniform charge density or a constant current and, in doing this, we get information of both the electric and magnetic self-interactions. We have chosen to apply here the method developed by Grats and Garcia in the ref. [15] which consists in describing the renormalised quantities using Riemannian coordinates, and in choosing the origin of the Riemannian frame at the centre of the geodesics. This method has been proved to be quite successful and, with our calculations, we generalize the work previously done by one of us in ref. [5].

The layout of this work is the following. In section 2, we calculate the self-energy and the self-force on a line source in the presence of a scalar-tensor cosmic string. We compare these expressions with the ones obtained in the General Relativity case. In the section 3, we summarize our main results.

\section{THE SELF-ENERGY AND THE SELF-FORCE ON A LINE SOURCE}

The solution of Einstein equations for a straight infinite cosmic string in the framework of the scalar-tensor theory was found, in the weak field approximation, by one of us [16]. The line element, in cylindrical coordinates, with the string placed along the $z$-axis, is given by

$$
\begin{aligned}
d s^{2}= & {\left[1+8 G_{0} \mu \alpha^{2}\left(\phi_{0}\right) \ln \left(R / R_{c}\right)\right] } \\
& \cdot\left(-d t^{2}+d z^{2}+d R^{2}+\beta^{2} R^{2} d \varphi^{2}\right),
\end{aligned}
$$

where $\beta^{2}=\left(1-8 G_{0} \mu\right)$. Here, $G_{0}$ is the $\phi_{0}$-dependent effective gravitational constant defined as $G_{0} \equiv G_{*} A^{2}\left(\phi_{0}\right)$ $\left(G_{*}\right.$ being some bare gravitational coupling constant, $A^{2}$ is an arbitrary function of the dilaton field $), \alpha^{2}\left(\phi_{0}\right)$ is a Post-Newtonian parameter which expresses the coupling between matter and the dilaton field and $\phi_{0}$ is the value 
of the dilaton field evaluated by solar systems experiments [17.

Since our problem is effectively two-dimensional, we will work with the ( $t=$ const, $z=$ const $)$ section of metric (11). For convenience, we perform the change of variables

$$
R=\frac{r^{\beta}}{\beta}
$$

in order to write the two-dimensional metric in conformal fashion,

$$
d s^{2}=\exp [-\Omega(r)]\left(d r^{2}+r^{2} d \varphi^{2}\right),
$$

where

$$
\Omega(r)=-\ln \left\{r^{2 \beta-2}\left[1+8 G_{0} \mu \alpha^{2}\left(\phi_{0}\right) \ln \left(\frac{r^{\beta}}{\beta R_{c}}\right)\right]\right\} .
$$

Consider now an infinitely long straight wire, placed parallel to the $z$-axis, at the position $\vec{r}^{\prime}=\left(r^{\prime}, \varphi^{\prime}\right)$, with uniform linear charge density $\lambda$. The corresponding three-dimensional charge density is

$$
\rho(\vec{r})=\lambda \frac{\delta\left(r-r^{\prime}\right) \delta\left(\varphi-\varphi^{\prime}\right)}{r}=\lambda \delta^{(2)}\left(\vec{r}-\vec{r}^{\prime}\right) .
$$

Given an arbitrary distribution of charge $\rho(\vec{r})$,the electrostatic energy is given by [18

$$
W=\frac{1}{2} \iint \rho(\vec{r}) G^{(3)}\left(\vec{r}, \vec{r}^{\prime}\right) \rho\left(\vec{r}^{\prime}\right) d^{3} r d^{3} r^{\prime},
$$

where $G^{(3)}\left(\vec{r}, \vec{r}^{\prime}\right)$ is the three-dimensional Laplacian Green function. In our case, the problem is effectively two-dimensional. So, the electrostatic energy per unit length is

$$
\frac{U_{\text {ele }}}{l}=\frac{1}{2} \iint \rho(\vec{r}) G_{d}^{(2)}\left(\vec{r}, \vec{r}^{\prime}\right) \rho\left(\vec{r}^{\prime}\right) d^{2} r d^{2} r^{\prime},
$$

where $G_{d}^{(2)}\left(\vec{r}, \vec{r}^{\prime}\right)$ is the two-dimensional Green function in the space described by metric (3).

By substituting (5) in (7) and taking the limit $\vec{r} \rightarrow \vec{r}^{\prime}$ the electrostatic energy per unit length is obtained as

$$
\frac{U_{e l e}}{l}=\left.\frac{\lambda^{2}}{2} G_{d}^{(2)}\left(\vec{r}^{\prime}, \vec{r}^{\prime}\right)\right|_{r e g},
$$

where $\left.G_{d}^{(2)}\left(\vec{r}^{\prime}, \vec{r}^{\prime}\right)\right|_{r e g}$ is the regularized Green function

$$
\left.G_{d}^{(2)}\left(\vec{r}^{\prime}, \vec{r}^{\prime}\right)\right|_{r e g}=\lim _{\vec{r} \rightarrow \vec{r}^{\prime}}\left[G_{d}^{(2)}\left(\vec{r}, \vec{r}^{\prime}\right)-G^{(2)}\left(\vec{r}, \vec{r}^{\prime}\right)\right] .
$$

Here, $G^{(2)}\left(\vec{r}, \vec{r}^{\prime}\right)$ is the Euclidean Green function, solution of the two-dimensional Poisson equation

$$
\Delta_{E} G^{(2)}\left(\vec{r}, \vec{r}^{\prime}\right)=-4 \pi \delta^{2}\left(\vec{r}-\vec{r}^{\prime}\right),
$$

where $\Delta_{E}$ is the two-dimensional Euclidean Laplacian.
The regularization, by extraction of the divergent part of the Green function, is necessary in order to obtain a finite result for the energy. On the other hand, this also guarantees that no finite self-energy survives in the absence of the cosmic string.

It has been shown by Grats and Garcia [15] that, since any two-dimensional metric can be put in the form given by eq. (3), the Laplacian Green function in the corresponding space can be expanded in terms of the geodesic distance $\sigma\left(\vec{r}, \vec{r}^{\prime}\right)$, such that

$$
\begin{aligned}
-G_{d}^{(2)}\left(\vec{r}, \vec{r}^{\prime}\right)= & \ln \left[2 \sigma\left(\vec{r}, \vec{r}^{\prime}\right)\right]+\Omega(\vec{r}) \\
& +(2 \sigma) \frac{t^{a} t^{b} \theta_{a b}}{24}+O\left(\sigma^{2}\right),
\end{aligned}
$$

where $2 \sigma\left(\vec{r}, \vec{r}^{\prime}\right)$ is the squared geodesic distance between the points $\vec{r}$ and $\vec{r}^{\prime}, t^{a}$ is the tangent vector to the geodesic at point $\vec{r}$, and the tensor $\theta_{a b}$ has the form

$$
\theta_{a b}=\nabla_{a} \Omega \nabla_{b} \Omega-\frac{1}{2} g_{a b} \nabla_{c} \Omega \nabla^{c} \Omega+\nabla_{a b}^{2} \Omega,
$$

where $\nabla_{a}$ is the covariant derivative and $g_{a b}$ is the metric tensor.

Since the Euclidean Green function can be written as

$$
G^{(2)}\left(\vec{r}, \vec{r}^{\prime}\right)=-\ln \left[2 \sigma\left(\vec{r}, \vec{r}^{\prime}\right)\right],
$$

it turns out that 15]

$$
\left.G_{d}^{(2)}\left(\vec{r}^{\prime}, \vec{r}^{\prime}\right)\right|_{r e g}=-\Omega\left(\vec{r}^{\prime}\right) .
$$

This equation shows explicitly that the self-energy appears from the geometry induced by the defect.

Using (14), (8), (4) and returning to the variable $R$, eq. (2), we get

$$
\begin{aligned}
\frac{U_{\text {ele }}}{l}= & \frac{\lambda^{2}}{2} \ln \left\{(\beta R)^{2-2 / \beta}\right. \\
& \left.\cdot\left[1+8 G_{0} \mu \alpha^{2}\left(\phi_{0}\right) \ln \left(\frac{R}{R_{c}}\right)\right]\right\}
\end{aligned}
$$

and

$$
\begin{aligned}
& \frac{\vec{F}_{\text {ele }}}{l}=-\vec{\nabla}\left(\frac{U_{\text {ele }}}{l}\right)=\frac{\lambda^{2}}{2 R} \\
& \cdot\left[2\left(\frac{1}{\beta}-1\right)-\frac{8 G_{0} \mu \alpha^{2}\left(\phi_{0}\right)}{1+8 G_{0} \mu \alpha^{2}\left(\phi_{0}\right) \ln \left(\frac{R}{R_{c}}\right)}\right] \widehat{r} .
\end{aligned}
$$

Notice that, when $\alpha^{2}\left(\phi_{0}\right) \rightarrow 0$, we recover the Einstein gravity result, equation (18), of reference [5].

Now, in order to study the magnetic self-force, we consider the infinitely long straight wire carrying the current density

$$
\vec{J}(\vec{r})=I \frac{\delta\left(r-r^{\prime}\right) \delta\left(\varphi-\varphi^{\prime}\right)}{r} \hat{z}=I \delta^{(2)}\left(\vec{r}-\vec{r}^{\prime}\right) r \hat{z} .
$$


The magnetic analogue of equation (17) is [18]

$$
\frac{U_{m a g}}{l}=\frac{1}{2 c^{2}} \iint \vec{J}(\vec{r}) \cdot \vec{J}\left(\vec{r}^{\prime}\right) G_{d}^{(2)}\left(\vec{r}, \vec{r}^{\prime}\right) d^{2} r d^{2} r^{\prime}
$$

which results in

$$
\frac{U_{m a g}}{l}=\left.\frac{I^{2}}{2 c^{2}} G_{d}^{(2)}\left(\vec{r}^{\prime}, \vec{r}^{\prime}\right)\right|_{r e g},
$$

analogous to equation (8).

As shown in [5], the magnetic self-force per unit length is given by

$$
\frac{\vec{F}_{m a g}}{l}=\vec{\nabla}\left(\frac{U_{m a g}}{l}\right) .
$$

Equations (20), (19) combined with (14) and (44) result in

$$
\begin{aligned}
& \frac{\vec{F}_{m a g}}{l}=-\frac{I^{2}}{2 c^{2} R} \\
& \cdot\left[2\left(\frac{1}{\beta}-1\right)-\frac{8 G_{0} \mu \alpha^{2}\left(\phi_{0}\right)}{1+8 G_{0} \mu \alpha^{2}\left(\phi_{0}\right) \ln \left(\frac{R}{R_{c}}\right)}\right] \widehat{r} .
\end{aligned}
$$

Comparing the expression for the magnetic self-force, eq. (21), with the one for the electric self-force, eq. (16), we see that they are proportional to each other:

$$
\frac{\vec{F}_{m a g}}{l}=-\frac{I^{2}}{c^{2} \lambda^{2}} \frac{\vec{F}_{\text {ele }}}{l} .
$$

Let us discuss now the behavior of the self-force. From equation (16) it is clear that $R$ can not be less than

$$
R_{\text {min }}=R_{c} \exp \left(-\frac{1}{8 G_{0} \mu \alpha^{2}\left(\phi_{0}\right)}\right) .
$$

In fact, writing (16) in terms of $R_{\text {min }}$, we get

$$
\frac{\vec{F}_{\text {ele }}}{l}=\frac{\lambda^{2}}{2 R}\left[2\left(\frac{1}{\beta}-1\right)-\frac{1}{\ln \left(\frac{R}{R_{\text {min }}}\right)}\right] \widehat{r} .
$$

This is in complete agreement with the metric (10) which makes sense only for $R>R_{\text {min }}$, as can be easily verified.

Notice that, for $R>>R_{\min }$ the behavior of both the electric and magnetic self-forces approaches that of the corresponding General relativity (GR) results 5 ]:

$$
\begin{aligned}
\frac{\vec{F}_{\text {ele }}}{l} & =\frac{\lambda^{2}}{R}\left(\frac{1}{\beta}-1\right) \\
\frac{\vec{F}_{\text {mag }}}{l} & =-\frac{I^{2}}{c^{2} R}\left(\frac{1}{\beta}-1\right) .
\end{aligned}
$$

This behavior is seen in Figure 1 for $x=R / R_{\min }>>1$.

Note in Figure 1 that the force changes its sign at some $R=R_{0}$ which can be obtained from eq. (24) with the condition $\vec{F}_{\text {ele }} / l=0$,

$$
R_{0}=R_{\text {min }} \exp \left[\frac{\beta}{2(1-\beta)}\right] \text {. }
$$

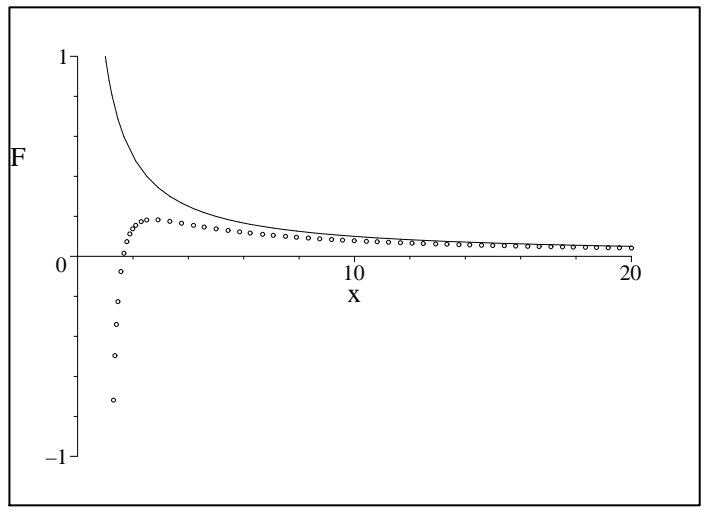

FIG. 1: The electric self-force per unit length $F$ as a function of $x=R / R_{\min }$ for $\beta=0.5$ and $\lambda^{2}=1$. The continuous line represents the GR result.

Differently from the GR result which is always repulsive, the Scalar-Tensor (ST) electric force is attractive in the range $R_{\min }<R<R_{0}$. Eq. (22) implies a similar analysis to $\frac{\vec{F}_{m a g}}{l}$, with inverted behavior: the magnetic force is attractive for $R>R_{0}$ and repulsive for $R_{\text {min }}<R<R_{0}$.

\section{CONCLUSIONS}

In ref. [20], one of us investigated the internal nature of ordinary cosmic vortices in some scalar-tensor extensions of gravity. Solutions were found for which the dilaton field condenses inside the vortex core. These solutions could be interpreted as raising the degeneracy between the eigenvalues of the effective stress-energy tensor, namely the energy per unit length $U$ and the tension $T$, by picking a privileged spacelike or timelike coordinate direction; in the latter case, a phase frequency threshold occured that is similar to what is found in ordinary neutral current-carrying cosmic strings. It has been found that the dilaton contribution for the equation of state, once averaged along the string worldsheet, vanishes, leading to an effective Nambu-Goto behavior of such a string network in cosmology, i.e. on very large scales. It has been shown also that, on small scales, the energy per unit length and tension depend on the string internal coordinates in such a way as to permit the existence of centrifugally supported equilibrium configuration, also known as vortons, whose stability, depending on the very short distance (unknown) physics, can lead to catastrophic consequences on the evolution of the Universe.

This statement is in a remarkable agreement with our present result. Indeed, in this work we determined the electric and magnetic self-interaction of line sources in the presence of a scalar-tensor cosmic string. When the source is placed away from the defect (long scales), the self-interaction is quite similar to the result obtained in the General Relativity framework. On the other hand, near the defect, the dilaton field introduces interesting 
new phenomena: a forbidden region $0<R<R_{\min }$ and an inversion of the electric (magnetic) self-force behavior, going from repulsive (attractive) in the far region to attractive (repulsive) in the near region.

Our main conclusion is that the coupling between a cosmic string and a scalar-tensor gravity is shown to induce effects along the string comparable to a current flow in the sense that the resulting effective stress-energy tensor eigenvalues, the energy per unit length $U$ and tension $T$, were no longer degenerate, due to the presence of the dilaton. Our model reproduces an Abrikosov flux tube coupled to a disclination in a condensed matter system 21]. We plan to continue our future works in this direction.

\section{Acknowledgments}

This work was partially supported by PRONEX/FAPESQ-PB, CNPq and CAPES (PROCAD).
[1] C. M. de Witt and B. S. de Witt, Physics 1 (1964) 3 ; W. G. Unruh, Proc. R. Soc. London A 348 (1976) 447.

[2] A. Vilenkin, Phys. Rev. D 20 (1979) 373.

[3] B. Linet, Phys. Rev. D 33 (1986) 1833.

[4] A. G. Smith, in Proceedings of Symposium on The Formation and Evolution of Cosmic String, edited by G. W. Gibbons, S. W. Hawking, and T. Vachaspati (Cambridge University Press, Cambridge, England, 1990).

[5] E. R. Bezerra de Mello, V. B. Bezerra, C. Furtado and F. Moraes, Phys. Rev. D 51 (1995) 7140.

[6] M. B. Green, J. H. Schwarz and E. Witten, Superstring Theory (Cambridge University Press, Cambridge, England, 1987).

[7] N. Arkani-Hamed, S. Dimopoulos and G. R. Dvali, Phys. Lett. B 429 (1998) 263; N. Arkani-Hamed, S. Dimopoulos and G. R. Dvali, Phys. Rev. D 59 (1999) 086004; I. Antoniadis, N. Arkani-Hamed, S. Dimopoulos and G. R. Dvali, Phys. Lett. B 436 (1998) 257.

[8] L. Randall and R. Sundrum, Phys. Rev. Lett. 83 (1999) 3370; L. Randall and R. Sundrum, Phys. Rev. Lett. 83 (1999) 4690.

[9] For a deeper discussion on this subject, we refer the reader to the book of Y. Fujii and Kei-Ichi Maeda, The Scalar-Tensor Theory of Gravitation (Cambridge Univ. Press, Cambridge 2003).

[10] S. Nussinov and R. Shrock, Phys. Rev. D 591999) 105002; E. A. Mirabelli, M. Perelstein and M. E. Peskin, Phys. Rev. Lett. 82 (1999) 2236; J. L. Hewett, Phys. Rev. Lett. 82 (1999) 4765 ; G. Shiu, R. Shrock and S. H. H. Tye, Phys. Lett. B 458 (1999) 274.

[11] S. Dimopoulos and G. Landsberg, Phys. Rev. Lett. 87 (2001) 161602; S. B. Giddings and S. Thomas, Phys. Rev.
D 65 (2002) 056010; S. Dimopoulos and R. Emparan, Phys. Lett. B 526 (2002) 393.

[12] J. L. Feng and A. D. Shapere, Phys. Rev. Lett. 88 (2002) 021303; L. Anchordoqui and H. Goldberg, Phys. Rev. D 65 (2002) 047502; R. Emparan, M. Masip and R. Rattazzi, Phys. Rev. D 65 (2002) 064023.

[13] N. Arkani-Hamed, S. Dimopoulos and G. R. Dvali, Phys. Rev. D 59 (1999) 086004; S. Cullen and M. Perelstein, Phys. Rev. Lett. 83 (1999) 268; V. D. Barger, T. Han, C. Kao and R. J. Zhang, Phys. Lett. B 461 (1999) 34; S. Hannestad and G. G. Raffelt, Phys. Rev. Lett. 88 (2002) 071301.

[14] M. Milgrom, Ap. J. 270, 365 (1983); R. H. Sanders, Mon. Not. Roy. Astron. Soc. 296 (1998) 1009; J. D. Bekenstein, "Relativistic gravitation theory for the MOND paradigm," arXiv:astro-ph/0403694.

[15] Yu. Grats and A. Garcia, Class Quantum Grav. 13 (1996) 189.

[16] M. E. X. Guimarães, Class. Quantum Grav. 14 (1997) 435.

[17] Th. Damour and K. Nordtvedt Jr., Phys. Rev. D 48 (1993) 3436.

[18] J. D. Jackson, Classical Electrodynamics, Wiley, New York, 1975.

[19] D. J. Griffiths, Introduction to Electrodynamics, 3rd edition, Prentice Hall, Upper Saddle River, 1999, page 518, equation (12.68).

[20] P. Peter, M. E. X. Guimarães and V. C. Andrade, Phys. Rev. D 67 (2003) 123509.

[21] C. Furtado, V. B. Bezerra and F. Moraes, Phys. Lett. A 289 (2001) 160. 